\title{
CÁlculo de Altitudes Científicas e SUA APLICAÇÃO NO REAJUSTAMENTO DA Rede AltimÉtrica de Alta Precisão do SISTEMA GEODÉSICO BRASILEIRO
}

\author{
Roberto Teixeira Luz \\ Instituto Brasileiro de Geografia e Estatística e \\ Universidade do Estado do Rio de Janeiro
}

\section{Resumo}

\begin{abstract}
A realização rigorosa de sistemas geodésicos de referência vertical deve se dar por intermédio de conjuntos de altitudes vinculadas ao campo da gravidade terrestre, isto é, as chamadas altitudes físicas, ou científicas. Somente estas refletem o paradigma de que "a água flui do ponto mais alto para o mais baixo". Neste artigo, são revistos os principais conceitos referentes aos sistemas geodésicos de referência vertical e às altitudes físicas, no contexto da preparação do primeiro ajustamento da Rede Altimétrica de Alta Precisão (RAAP) do Sistema Geodésico Brasileiro considerando diferenças de geopotencial, isto é, desníveis da RAAP corrigidos com dados gravimétricos reais. São apresentados e discutidos os resultados de um ensaio com a rede considerada em um dos ajustamentos gerais anteriores da RAAP.
\end{abstract}

Palavras-chave: números geopotenciais; altitudes físicas; nivelamento; gravidade; datum vertical; SIRGAS.

\begin{abstract}
The rigorous realization of vertical geodetic reference systems must take place via sets of heights linked to the Earth's gravity field, i.e. the so-called physical or scientific heights. Only these reflect the paradigm that 'water flows from the highest point to the lowest one'. In this paper, the main concepts related to vertical geodetic reference systems and physical heights are reviewed in the context of the preparation of the first adjustment of High Precision Altimetric Network (RAAP) of the Brazilian Geodetic System considering geopotential differences, that is, RAAP's height differences corrected with real gravity data. The results of a trial adjustment are presented and discussed, considering the network selected for one of the previous general adjustments of RAAP.
\end{abstract}

Key words: geopotential numbers; physical heights; leveling; gravity; vertical datum; SIRGAS.

força da gravidade modela e condiciona toda a vida na Terra. É a gravidade que mantém a atmosfera junto ao planeta, é ela que também estabelece a orientação espacial de todos os seres vivos no planeta e, no contexto da Geodésia, serve de referência a grande parte das observações - o próprio significado de conceitos básicos como "horizontal" e "vertical" reflete a importância do campo da gravidade e relaciona-se, implicitamente, a seus elementos geométricos básicos, isto é, suas linhas de força e superfícies equipotenciais (LUZ, 2008). Da mesma forma, o conceito de altitude física (ou científica) possui um inexorável vínculo com o campo da gravidade terrestre, e o cálculo de altitudes de referência - como aquelas vinculadas às Referências de Nível (RRNN) do Sistema Geodésico Brasileiro (SGB) - deve necessariamente incorporar dados gravimétricos. O advento dos modernos sistemas de navegação e posi- 
cionamento por satélites (Global Navigation Satellite Systems, GNSS), dentre os quais situam-se o GPS e o GLONASS, representou uma inesperada ênfase daquele vínculo, já que as altitudes elipsoidais resultantes dos GNSS são puramente geométricas, totalmente desvinculadas do campo da gravidade. Em função desta característica, sua aplicação direta no posicionamento vertical raramente é aceitável, requerendo correções adicionais, geralmente obtidas a partir dos chamados mapas geoidais, que permitem a transformação das altitudes geométricas em altitudes físicas. Estas características são exemplificadas na Figura 1, em que se representa a importância do correto posicionamento vertical das barragens "em cascata" ao longo de um rio.

Assim, nos contextos técnicos e científicos em que seja necessária precisão submétrica no posicionamento vertical, a utilização do conceito de altitude deve ser acompanhada pela identificação do tipo específico de altitude empregada. Em contraposição às já mencionadas altitudes geométricas (elipsoidais), resultantes da aplicação dos GNSS, as altitudes físicas são caracterizadas por respeitarem e refletirem o comportamento hidráulico. São contadas a partir de uma certa superfície de nível (equipotencial) do campo da gravidade, materializada de forma aproximada pelo nível médio do mar, e seu cálculo se dá por meio da associação de informação gravimétrica aos desníveis observados na operação de nivelamento geométrico de alta precisão.

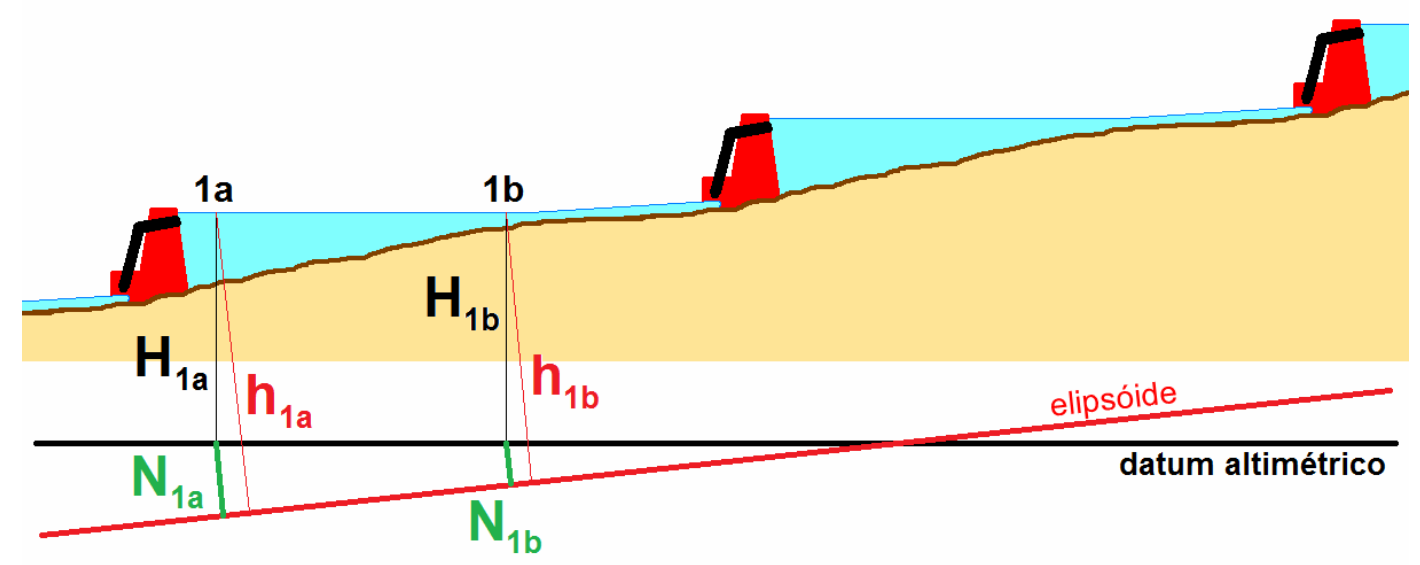

Figura 1. Representação esquemática das altitudes físicas $(H)$ e geométricas $(h)$ utilizadas no posicionamento vertical de barragens "em cascata" (contexto em que se busca otimizar a geração do sistema, maximizando o nível d'água em cada barragem sem afogar os geradores a montante), ressaltando-se a necessidade de corrigir as altitudes geométricas com as alturas geoidais $(\mathrm{N})$ de forma a compatibilizá-las com as altitudes físicas do sistema altimétrico nacional.

Neste artigo, são revistos os principais conceitos referentes aos sistemas geodésicos de referência vertical e às altitudes físicas, no contexto da preparação do primeiro ajustamento da Rede Altimétrica de Alta Precisão (RAAP) do SGB considerando diferenças de geopotencial, isto é, desníveis corrigidos com dados gravimétricos reais. São apresentados e discutidos os resultados de um ensaio com a rede considerada no Ajustamento Altimétrico Global Preliminar (AAGP, conforme LUZ \& GUIMARÃES, 2001). 


\section{Geopotencial}

Considerando a necessidade de incluir a força da gravidade (g) na descrição de um sistema de referência para o posicionamento vertical, a complexidade decorrente de sua natureza vetorial é eliminada observando-se a condição de se tratar de um campo conservativo (VANÍCEK \& KRAKIWSKY, 1986). Sob tal condição, o campo vetorial g pode ser adequadamente descrito pelo campo escalar $\mathrm{W}$ tal que

$$
g=\operatorname{grad} W=\underline{\nabla} W=\frac{\partial W}{\partial x} \underline{i}+\frac{\partial W}{\partial y} j+\frac{\partial W}{\partial z} \underline{k}
$$

sendo $i, j$ e $k$ os vetores unitários na direção dos eixos $X, Y$ e $Z$, respectivamente. O campo escalar $W$ é denominado potencial da gravidade, ou geopotencial, resultado da composição dos potenciais gravitacional $W_{G}$ e centrífugo $W_{C}$.

O geopotencial tem grande importância na Geodésia, remetendo aos elementos geométricos básicos mencionados anteriormente. O mais intuitivo desses elementos é a família de superfícies em que o geopotencial é constante - isto é, as superfícies equipotenciais, que "representam o plano horizontal local em relação ao qual os instrumentos geodésicos são posicionados" (VANíCEK, 1976, p. 54).

Tais aspectos geométricos do geopotencial são usualmente discutidos a partir da expressão de sua derivada sob a forma do produto escalar (HEISKANEN \& MORITZ, 1967, p. 48):

$$
d W=\frac{\partial W}{\partial x} d x+\frac{\partial W}{\partial y} d y+\frac{\partial W}{\partial z} d z=g \cdot \underline{\mathbf{d u}}
$$

sendo uu um vetor arbitrário, de componentes ( $d x, d y, d z)$.

Desta expressão resulta que o vetor gravidade é sempre ortogonal às superfícies equipotenciais - já que, para du sobre uma equipotencial qualquer, o trabalho dW é nulo. Daí surge o conceito de linhas de força do geopotencial, ou linhas de prumo: curvas tangentes ao vetor gravidade em cada ponto, ao longo das quais é máxima a variação da magnitude da gravidade.

Linhas de força e superfícies equipotenciais materializam, portanto, os conceitos "vertical" e "horizontal" - na verdade, a definição geodésica de linha vertical é equivalente àquela definição de linha de força, e as equipotenciais são também chamadas superfícies de nível (TORGE, 2001).

Também da equação (2), com $\underline{\mathbf{d u}}$ agora orientado contrariamente a g, resulta a importante expressão que relaciona a diferença de geopotencial $d W$ entre duas equipotenciais à distância infinitesimal $d H$ que as separa (id., p. 58):

$$
d W=-g d H
$$


Esta expressão provê o arcabouço conceitual para a obtenção de diferenças de geopotencial a partir de informações derivadas de levantamentos geodésicos ( $d H$ e $g$, provenientes, respectivamente, de nivelamento geométrico e gravimetria).

Assim, apesar das dificuldades relacionadas à distribuição irregular de massas na crosta terrestre, às variações temporais relacionadas aos movimentos relativos entre Terra, Lua e Sol, e a outras influências, o geopotencial é a opção natural para a parametrização do posicionamento vertical (TORGE, 2001). Em seguida, é necessário discutir a seleção de uma das superfícies equipotenciais como referência vertical.

\section{Geoide}

A superfície dos oceanos estabelece um claro limite ao domínio espacial das atividades humanas. Além disso, ocupando mais de $70 \%$ da superfície terrestre, materializa a mais abrangente aproximação de uma superfície equipotencial do campo da gravidade (VANÍCEK \& KRAKIWSKY, 1986). Este fato justificou a proposição daquela superfície, em meados do século 19, como referência para a representação geométrica da superfície terrestre por J. C. F. Gauss (TORGE, 2001; GRAFAREND, 1994). Posteriormente, esta superfície foi nomeada "geoide" por J. B. Listing, conforme os mesmos autores.

No entanto, a "superfície oceânica" de Gauss e Listing corresponde a oceanos idealizados, homogêneos e em repouso, sujeitos apenas à força da gravidade (TORGE, 2001). Usualmente, as variações de alta frequência, como ondas, marés astronômicas e efeitos meteorológicos, eram eliminadas ou minimizadas por meio da promediação das observações de nível do mar em períodos suficientemente longos. O nível médio do mar (NMM) assim obtido era tomado como base natural para a materialização da superfície de referência vertical das redes geodésicas clássicas em todo o mundo (TORGE, 2001).

Todavia, mesmo nas mais longas séries temporais, o NMM ainda apresenta desvios em relação à superfície equipotencial, originados, por exemplo, do efeito termoestérico (diferença volumétrica) entre as águas tropicais e polares (VANÍCEK \& KRAKIWSKY, 1986). Tais desvios alcançam +/-2m, globalmente, e são denominados topografia do NMM (TNMM), ou topografia dinâmica dos oceanos (dynamic ocean topography, DOT).

O conceito de TNMM é essencial para uma definição rigorosa do geoide, eliminando a subjetividade implícita na proposta original de Gauss e Listing. Tal definição rigorosa contribui para a realização da referência vertical global de forma consistente com os níveis de precisão já alcançados pelas modernas técnicas geodésicas, no contexto dos recentes esforços da Associação Internacional de Geodésia (IAG, 2015a).

Revisões detalhadas do assunto são feitas, por exemplo, por Heck (2004) ou Sánchez (2012). No contexto do presente artigo, é suficiente destacar que os resultados da Rede Maregráfica Permanente para Geodésia (RMPG) ainda não são aplicáveis como injunções no ajustamento da RAAP, em função da necessidade de uma correta caracterização da TNMM no litoral brasileiro 
(IBGE, 2013, p. 37). Um exemplo extremo dos impactos da utilização de múltiplas estações maregráficas no estabelecimento de um datum vertical é a distorção norte-sul de até $1,5 \mathrm{~m}$ das altitudes australianas, cujo "datum de altitudes da Austrália" (AHD) foi estabelecido com informações de 30 estações maregráficas (FEATHERSTONE et al., 2011). Por outro lado, esse tipo de distorção não ocorre no SGB, já que o Datum Vertical Brasileiro em Imbituba (DVB-I) foi definido com base em uma única estação maregráfica (ALENCAR, 1990).

A seguir, são revistos resumidamente os principais tipos de altitudes físicas, conforme apresentados por Luz (2008).

\section{Altitudes Físicas}

Nas seções anteriores, os conceitos de geopotencial e geoide foram apresentados, respectivamente, como a grandeza que naturalmente parametriza o posicionamento vertical e a correspondente superfície de referência - reforçando a já mencionada condição de que sistemas geodésicos de referência vertical rigorosos devem ser realizados (materializados) por conjuntos de altitudes vinculadas ao campo da gravidade terrestre. Tais altitudes físicas $(H)$ atendem a paradigmas instintivos que relacionam conceitos como "alto" e "baixo" ao fluxo de água (TORGE, 2001). A elas contrapõem-se as altitudes referidas a modelos elipsoidais, dotadas de significado puramente geométrico. Enquanto as primeiras são obtidas por nivelamento geométrico associado a observações gravimétricas, as altitudes geométricas (h) resultam diretamente da aplicação das técnicas espaciais de posicionamento tridimensional - tais como aquelas associadas aos GNSS.

Assim, o geopotencial é a base para definição de coordenadas verticais. No entanto, o valor do geopotencial no geoide não é nulo. Para de fato referir a coordenada vertical ao geoide, a IAG recomendou, em 1954, a adoção do chamado número geopotencial C, definido como a diferença entre os geopotenciais no geoide e no ponto considerado. A partir da equação (3):

$$
C_{B}=W_{0}-W_{B}=\int_{0}^{B} g d H \approx \sum_{i=1}^{K}\left(g_{m}^{o b s} \Delta H^{o b s}\right)
$$

sendo $g_{m}{ }^{o b s}$ a média dos valores observados da gravidade em cada par de pontos nivelados (seção), $\Delta H^{\text {obs }}$ o respectivo desnível observado, e $K$ o número total de seções (TORGE, 2001). Na prática, é preferível o cálculo das diferenças de geopotencial $\Delta C_{A B}$, desde que a distância entre os pontos A e B seja suficientemente pequena:

$$
\Delta C_{A B}=C_{B}-C_{A}=-\left(W_{B}-W_{A}\right) \approx g_{A B}^{o b s} \Delta H_{A B}^{o b s}
$$

O ajustamento destas diferenças de geopotencial, adequadamente injuncionado, resulta nos 
números geopotenciais. A Figura 2 ilustra estes e os próximos conceitos.

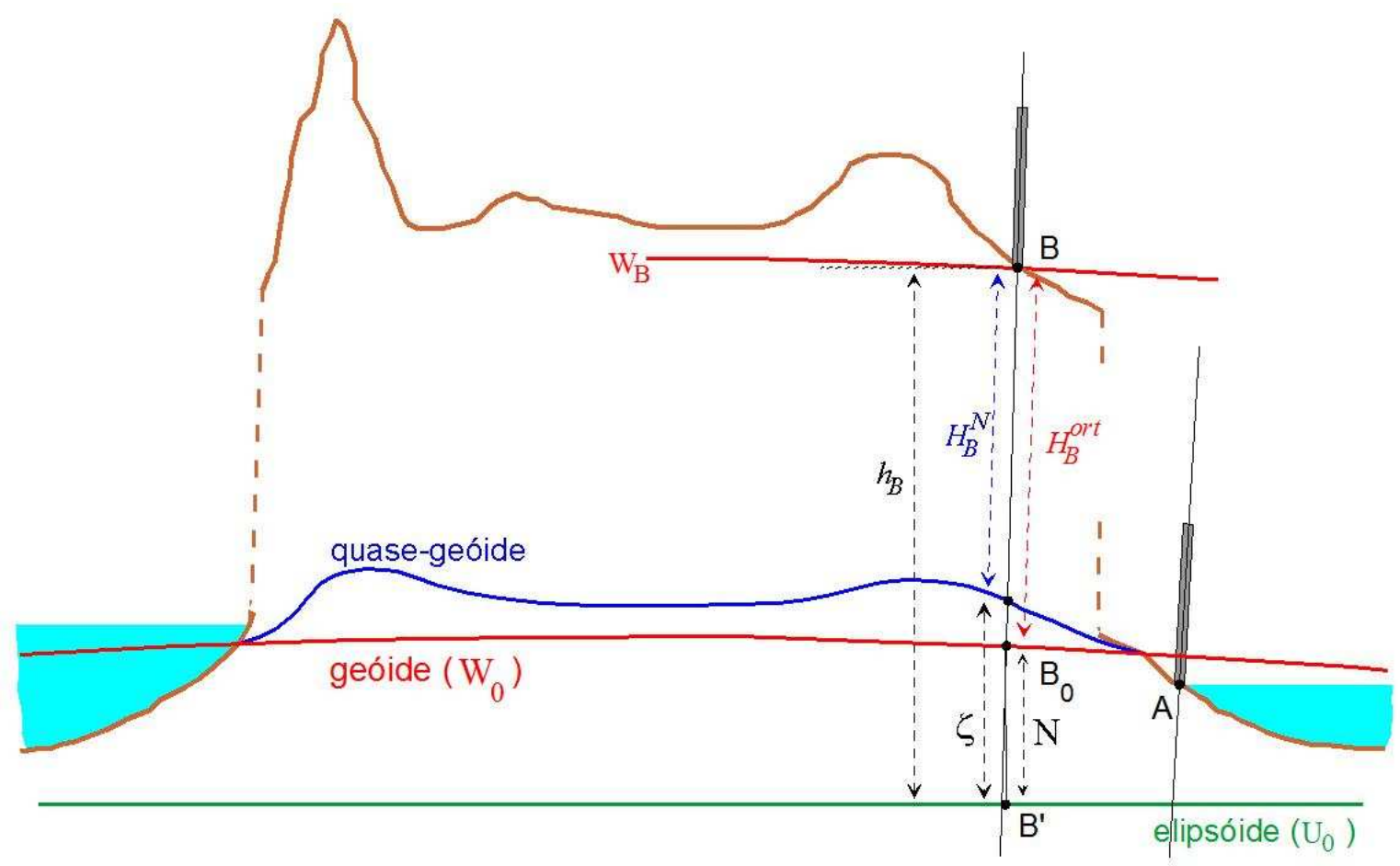

Figura 2. Elementos envolvidos na definição das altitudes físicas. Fonte: Luz (2008).

Uma das dificuldades para a adoção dos números geopotenciais como descritores rigorosos da posição vertical é a unidade em que são expressos $\left(\mathrm{m}^{2} / \mathrm{s}^{2}\right)$. Este problema pode ser contornado com a simples divisão de $C$ por valores de gravidade adequadamente selecionados, considerando variados critérios, resultando em diferentes tipos de altitudes físicas. Todas estas altitudes mantêm a importante característica de respeitar o significado intrínseco de altitude, refletindo o paradigma de que "a água flui do ponto mais alto para o mais baixo".

Classicamente, o principal tipo é a altitude ortométrica $\left(H^{\text {ort }}\right)$, definida como a distância do geoide ao ponto, sobre a linha vertical. Integrando-se, entre o geoide e a superfície física, a diferença entre os geopotenciais de duas superfícies equipotenciais separadas por uma distância infinitesimal (eq. 3), obtém-se (TORGE, 2001, p. 82):

$$
C_{B}=\int g d H=g_{m} \int d H \quad \ldots \quad H_{B}^{\text {ort }}=\frac{C_{B}}{g_{m}}
$$

sendo $g_{m}$ o valor médio da gravidade entre o geoide e a superfície física, ao longo da vertical $\left(B_{0}\right.$ e $B$, na Figura 2). 
A diferença entre as altitudes elipsoidal e ortométrica é a altura geoidal:

$$
N_{B} \approx h_{B}-H_{B}^{o r t}
$$

O efeito da não colinearidade de $H$ e $h$ é desprezível. O caso extremo de uma altitude de 10 km e um desvio da vertical de 1' (ângulo entre a normal ao elipsoide e a vertical), implica em um erro menor que $1 \mathrm{~mm}$ (JEKELI, 2000).

A determinação rigorosa das altitudes ortométricas ainda não é possível, em função da impossibilidade atual de se conhecer, com suficiente resolução, a distribuição de densidades - e, consequentemente, o valor médio da gravidade (FREITAS \& BLITZKOW, 1999) - no interior da crosta. Valores aproximados desse tipo de altitude resultam da aplicação de modelos simplificados da distribuição de densidades litosféricas. Por exemplo, o chamado gradiente de PoincaréPrey, $-0,0848 \mathrm{miligal} / \mathrm{m}$, surge da combinação da densidade crustal média $(2,67 \mathrm{~g} / \mathrm{cm} 3) \mathrm{com}$ o gradiente vertical da gravidade normal, -0,3086 miligal/m (GEMAEL, 2002). Aplicando-se o gradiente de Poincaré-Prey para reduzir a gravidade observada na superfície física $\left(g^{\text {obs }}\right)$ ao ponto médio $\left(H^{n i v} / 2\right)$ entre esta e o geoide, obtém-se um valor aproximado $\left(g_{m}{ }^{P P}\right)$ da gravidade média $g_{m}$, usado nas altitudes de Helmert $\left(H^{H}\right)$ :

$$
H^{H}=\frac{C}{g_{m}^{P P}}=\frac{C}{g^{o b s}+0,0424 H^{n i v}}
$$

Jekeli (2000) menciona a necessidade de um processo iterativo nesta expressão, substituindo $H^{\text {niv }}$ pelo valor calculado de $H^{H}$ até sua convergência.

No caso do sistema vertical da América do Norte (NAVD-88), as diferenças de geopotencial são ajustadas e, com base nos números geopotenciais resultantes, são calculados dois tipos de altitude, sendo uma delas a ortométrica de Helmert (ZILKOSKI et al., 1992).

Hwang e Hsiao (2003), para a rede fundamental de Taiwan (1010 RRNN, $2.000 \mathrm{~km}$ ), utilizaram um modelo de variações laterais da densidade em malha regular de 5'. Neste modelo, os valores situam-se entre menos de $2,0 \mathrm{~g} / \mathrm{cm} 3$ e $3,08 \mathrm{~g} / \mathrm{cm} 3$, respectivamente correspondentes às planícies costeiras e ao maciço montanhoso na parte central do país. A correção de Helmert original foi comparada com diferentes modificações, considerando variações das densidades e dos gradientes das anomalias da gravidade. Seus resultados mostraram que as variações de densidade não introduzem diferenças significativas, em relação à correção de Helmert original. Por outro lado, diferenças de até $15 \mathrm{~cm}$ surgem da utilização dos gradientes das anomalias da gravidade para altitudes acima de $3.500 \mathrm{~m}$. Para os circuitos que incluem as linhas com essas variações de altitude, o erro de fechamento foi significativamente melhorado, com qualquer uma das versões da correção de Helmert, em relação aos desníveis não corrigidos - p. ex., de 11,8 cm para 2,2 cm, e de $6,6 \mathrm{~cm}$ para $1,3 \mathrm{~cm}$.

Além da discussão sobre a viabilidade e exatidão de sua obtenção numérica, as altitudes or- 
tométricas caracterizam-se por não tornar clara a condição de que as superfícies equipotenciais são superfícies de nível - com elas, diferentes pontos de uma única superfície equipotencial podem possuir valores diferentes de altitude no sentido puramente geométrico. Para melhor abordar a questão, basta lembrar do não paralelismo das equipotenciais, que resulta na interessante situação (TORGE, 2001) em que duas equipotenciais separadas por 100 m no Equador mostram, nos polos, uma separação de apenas $99,5 \mathrm{~m}$. Isso leva a um importante questionamento (LUZ, 2008): seria necessário rever a conceituação de altitude, explicitando a necessidade de obedecer à condição de nível das superfícies equipotenciais? Em outras palavras, o significado físico das altitudes não seria mais importante que sua interpretação geométrica?

Da tentativa de contornar o problema da obtenção da gravidade média $g_{m}$, substituindo-a por outros valores, surgem outros tipos de altitudes físicas. A seguir são discutidos os principais desses tipos - uma revisão abrangente pode ser encontrada em Sánchez (2005).

Para as altitudes dinâmicas $\left(H^{D}\right)$, consideram-se valores constantes para a gravidade de referência, escolhidos arbitrariamente - por exemplo, para o Brasil poderia ser escolhida a média dos valores de gravidade real na superfície física, ou o valor de gravidade normal para a latitude média do país, ou ainda um valor que minimizasse as diferenças na região do Datum de Imbituba ou as diferenças em todos os marégrafos:

$$
H^{D}=\frac{C}{g^{\text {arbitr }}}
$$

Este tipo de altitude é ideal para estudos hidráulicos e hidrológicos, pois garante valores iguais de altitude para pontos sobre a mesma superfície equipotencial. Foi adotado, por exemplo, no sistema vertical específico dos Grandes Lagos (IGLD-85), na América do Norte, com valor de 9,806199 m/s2 no denominador (CC-GL-BHHD, 1995, p. 8). Zilkoski et al. (1992) afirmam que NAVD-88 e IGLD-85 são equivalentes, já que, em cada RRNN comum aos dois sistemas, os respectivos números geopotenciais são iguais.

No caso da América do Sul, o monitoramento do Aqüífero Guarani constitui um exemplo de possível aplicação das altitudes dinâmicas, em função das necessidades hidrológicas e de sua grande extensão.

Por outro lado, Torge (2001) menciona que a principal desvantagem das altitudes dinâmicas origina-se das diferenças relativamente grandes em relação às altitudes niveladas.

Para as altitudes normais $\left(H^{N}\right)$, substitui-se (FREITAS \& BLITZKOW, 1999) o valor médio da gravidade real $g_{m}$ pelo equivalente da gravidade normal, $\gamma_{m}$ :

$$
H^{N}=\frac{C}{\gamma_{m}}
$$

Isso faz com que as altitudes normais não se refiram propriamente ao geoide, mas sim a 
uma superfície próxima a ele - denominada, por isso, quase-geoide. À separação entre quasegeoide e elipsóide, contada a partir deste ao longo da normal (Figura 2), dá-se o nome de anoma-

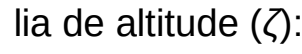

$$
\zeta \approx h-H^{N}=N-\frac{g_{m}-\gamma_{m}}{\gamma_{m}} H^{o r t} \frac{\Delta g_{B}{ }^{o r t}}{\gamma_{m}}
$$

sendo $\Delta g_{B}^{\text {ort }}$ a anomalia Bouguer.

Cabe ressaltar que a interpretação original da anomalia de altitude diz respeito à separação entre o teluróide e a superfície física, no contexto da teoria de Molodensky (e.g., TORGE, 2001).

O valor médio da gravidade normal, ao longo da linha vertical, para a latitude $\varphi$ e a altitude normal $H^{N}$, é dado por (HEISKANEN \& MORITZ, 1967, p. 170):

$$
\gamma_{m}^{v}=\gamma_{0}\left[1-\frac{H^{N}}{a}\left(1+\alpha+m-2 \alpha \operatorname{sen}^{2} \varphi\right)+\left(\frac{H^{N}}{a}\right)^{2}\right]
$$

sendo a, m, $\alpha$ e $y_{0}$, parâmetros vinculados ao sistema geodésico de referência (SGR).

Assim, a altitude normal é obtida, com precisão melhor que 0,01 mm, após poucas iterações - comumente, de três a cinco.

Por fim, no caso da inexistência de informação gravimétrica associada aos desníveis, um significado físico mínimo pode ser dado às altitudes resultantes por meio da correção normal ortométrica $\delta H^{\text {Nort }}$ (BOWIE \& AVERS, 1914, p. 52):

$$
\delta H^{\text {Nort }} \approx-2 \alpha_{1} H_{m} \Delta \varphi \operatorname{sen}\left(2 \varphi_{m}\right)\left[1+\left(\alpha_{1}-2 \alpha_{2} / \alpha_{1}\right) \cos \left(2 \varphi_{m}\right)\right]
$$

sendo $H_{m}$ a média das altitudes niveladas dos extremos da seção, $\Delta \varphi$ a correspondente diferença de latitudes, $\varphi$ m a média das mesmas, e os parâmetros $\alpha_{1}$ e $\alpha_{2}$ da fórmula de Helmert para a gravidade normal.

Para uma seção de nivelamento, em condições brasileiras comuns, o valor dessa correção ultrapassa facilmente $1 \mathrm{~mm}$. Para redes verticais de âmbito continental em que não se associam valores de gravidade, como a brasileira, é imprescindível, portanto, a aplicação da correção normal-ortométrica. 


\section{Altitudes SIRGAS}

Criado em 1993 com o objetivo inicial de unificar os sistemas geodésicos planimétricos do continente sul-americano, o Projeto SIRGAS alcançou seus primeiros resultados em 1997. Nesse ano, foram divulgadas as coordenadas da Rede de Referência GPS estabelecida em 1995, com estações em quase todos os países da região (IBGE, 1997). Com base no sucesso alcançado, o Projeto ampliou seus objetivos, visando também contemplar o complexo problema da definição de um Datum Vertical único para a América do Sul.

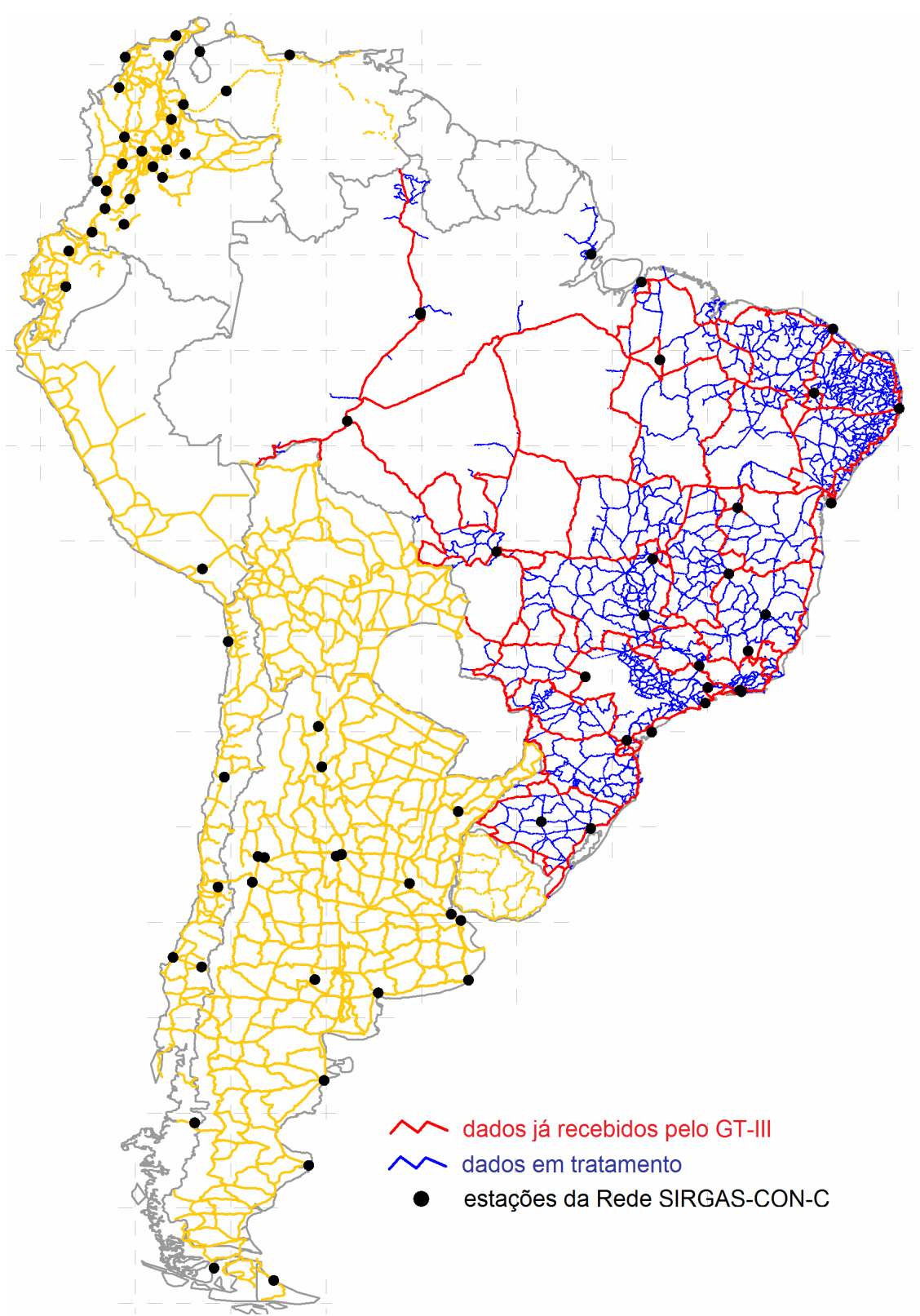

Figura 3. Redes de nivelamento sul-americanas e estações permanentes GNSS. Fonte: adaptado de SIRGAS (2011). 
Para isso, no mesmo ano foi criado o Grupo de Trabalho sobre Datum Vertical (GT-III). As primeiras recomendações do GT-IIl expressavam que (DREWES et al., 2002b):

a) o futuro DVSIRGAS (Datum Vertical SIRGAS) seria definido através de dois conjuntos de altitudes - geométricas e físicas --, bem como pelas respectivas taxas de variação temporal ("velocidades");

b) o futuro DVSIRGAS seria materializado através de uma rede de estações geodésicas determinadas com GPS, nivelamento geométrico e gravimetria, baseada nas estações da Rede de Referência de 1995 e estendida com estações nas fronteiras niveláveis entre os países sulamericanos e nas suas principais estações maregráficas (Figura 3); e

c) os países deveriam iniciar a organização e o levantamento dos dados necessários ao cálculo dos números geopotenciais, os quais são elementos intermediários para a obtenção das altitudes físicas - como discutido na próxima seção.

É importante destacar que a "materialização geopotencial" do DVSIRGAS, i.e., a determinação das altitudes físicas, não será feita a partir de observações maregráficas — pelo menos a princípio, de acordo com as recomendações e resoluções estabelecidas até o presente. As estações da Rede SIRGAS nas estações maregráficas servirão apenas para vincular ao novo datum os referenciais existentes. O GT-III recomendou aos países membros do SIRGAS que as altitudes físicas SIRGAS sejam do tipo normal, isto é, obtidas através da razão entre os números geopotenciais e o valor teórico ("normal") da gravidade. Nesse contexto, surge o problema da obtenção do valor do potencial do geoide, conhecido por $W_{0}$, assunto que vem sendo objeto de intensa pesquisa e discussão internacional (e.g., BOSCH, 2002; BURŠA et al., 2002; SJÖBERG, 2013), que culminaram com a recente recomendação de convenções associadas ao Sistema Internacional de Referência para Altitudes (IHRS, International Height Reference System), incluindo o valor de $W_{0}$ (IAG, 2015b).

\section{Altitudes brasileiras}

O estabelecimento da Rede Altimétrica de Alta Precisão (RAAP), sob coordenação inicial do então Conselho Nacional de Geografia (CNG), teve início em 1945, no extremo sul do Brasil (ALENCAR, 1990). Daí, a RAAP progrediu inicialmente em direção ao nordeste do país, através das regiões litorâneas de maior desenvolvimento econômico e demográfico, chegando também à recém estabelecida capital, Brasília (Figura 4). A partir da década de 1970, a implantação da RAAP foi direcionada ao interior do país, à substituição das redes de outras instituições e à reconstituição dos trechos destruídos.

Até 1959, as altitudes da RAAP referiram-se provisoriamente ao Datum de Torres, no Rio Grande do Sul (RS), materializado pelo NMM obtido de observações maregráficas no período de fevereiro de 1919 a fevereiro de 1920 (ALENCAR, 1968). Em 1959 foi concluído o terceiro ajustamento da RAAP, quando então suas altitudes passaram a referir-se ao Datum de Imbituba. Este foi definido com base na média dos NMMs anuais, entre 1949 e 1957, resultantes das informa- 
ções coletadas pelo Inter American Geodetic Survey (IAGS) na estação maregráfica instalada em 1948, como parte de um extenso programa de cooperação com diversas instituições brasileiras e também com outros países latino-americanos.

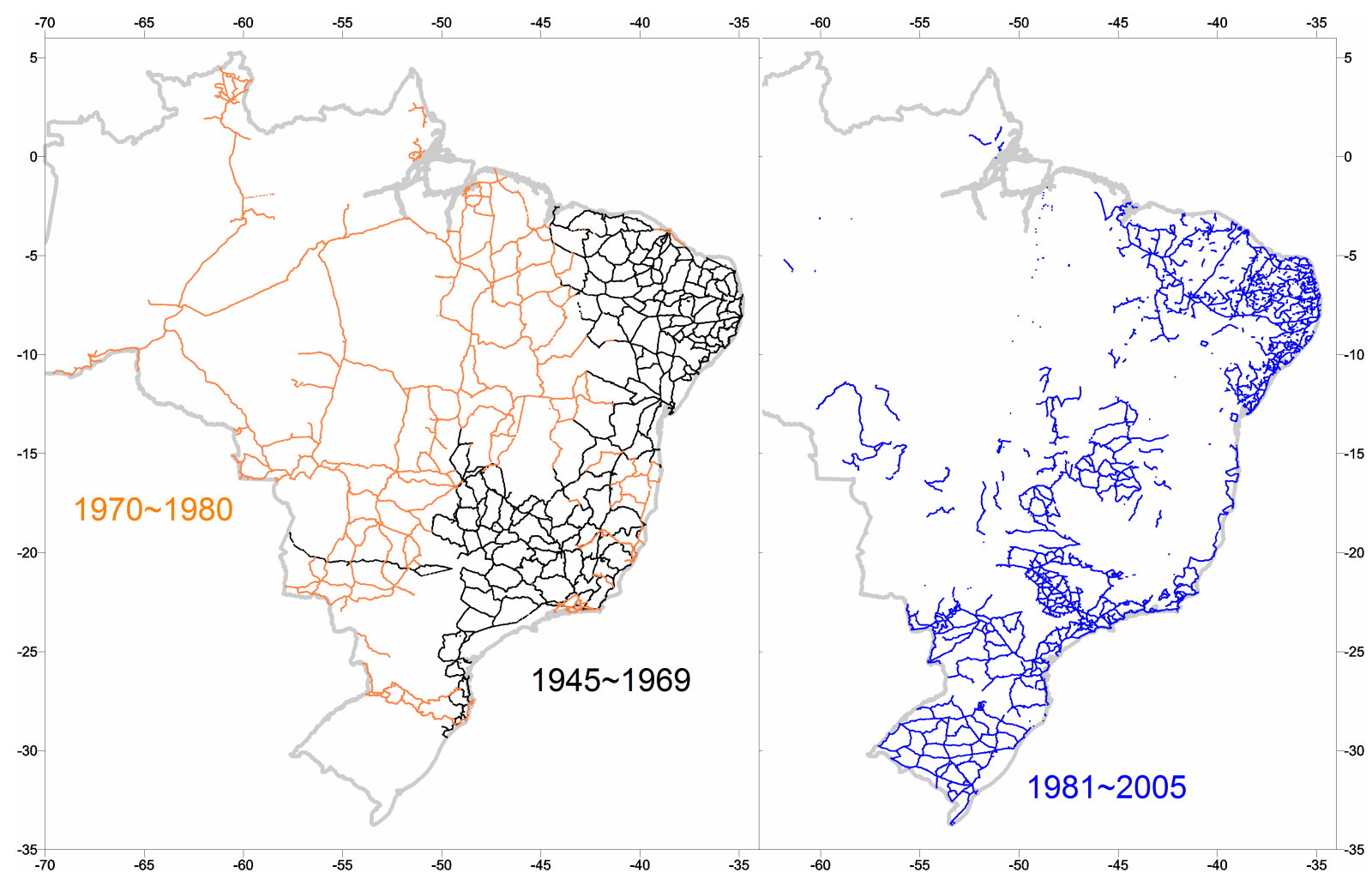

Figura 4. Evolução temporal da RAAP (Fonte: Luz, 2008). À esquerda: RRNN estabelecidas de 1945 a 1969 , em preto - progredindo do litoral sul em direção nordeste - e de 1970 a 1980, em laranja, quando se iniciou a interiorização do nivelamento. À direita, RRNN estabelecidas de 1981 a 2005, em azul, para reconstituição da rede e atendimento às áreas não cobertas anteriormente.

A porção da RAAP no estado do Amapá refere-se a um datum local, pois não é possível cruzar o Rio Amazonas com nivelamento geométrico nesse trecho. O Datum Vertical Brasileiro em Santana (DVB-S) foi definido por observações de nível do mar no porto de mesmo nome, entre 1957 e 1958.

As medições de nivelamento geométrico para estabelecimento da RAAP foram realizadas, em sua grande maioria, com níveis Wild N3, tripés rígidos e miras invar de dupla graduação, segundo os procedimentos usuais para obtenção de alta precisão (BESERRA, 1948; IBGE, 1983, 1985):

a. nivelamento e contra-nivelamento (N/CN) usualmente feitos em sequência, não necessariamente nessa ordem, mas sempre com alternância de operadores. Até 1983, a diferença entre N e CN devia atender à tolerância de 4 mm $[\mathrm{km}]^{1 / 2}$. Em 1983, essa tolerância foi redu- 
zida para $3 \mathrm{~mm}[\mathrm{~km}]^{1 / 2}$, referente aos levantamentos "de alta precisão" (IBGE, 1983);

b. novas linhas são vinculadas às existentes somente em RRNN cuja estabilidade relativa seja confirmada mediante a chamada verificação de abalo, i. e., renivelamento de pelo menos duas seções antigas. A tolerância para as diferenças é a mesma utilizada para a diferença N/CN de uma seção;

c. miras lidas usualmente na sequência natural do trabalho (esquerda e direita de ré, esquerda e direita de vante, RRVV), com a alternância da mira utilizada à ré e à vante para eliminação do erro de índice;

d. observação realizada usualmente nas primeiras horas do dia, até o momento em que os efeitos do aumento da temperatura começam a prejudicar a horizontalização do nível de boIha e a leitura das miras. Eventualmente os trabalhos foram também realizados nos finais de tarde;

e. erro de colimação determinado diariamente, no início dos trabalhos, com limite máximo de $0,03 \mathrm{~mm} / \mathrm{m}$;

f. leitura mínima de $30 \mathrm{~cm}$, a fim de evitar os efeitos da refração nas camadas de ar próximas ao solo;

g. comprimento das visadas de até 120 m (ALENCAR, 1968), sendo utilizado atualmente o limite de $60 \mathrm{~m}$;

h. visadas aproximadamente iguais a ré e a vante $(R / V)$, com tolerância de $3 \mathrm{~m}$ para a diferença entre os somatórios de seção, a fim de evitar a necessidade de aplicação das correções de colimação, esfericidade e refração; e

i. controles de qualidade das observações (constante da mira, intervalos de estádia e comprimentos R/V) realizado pelo anotador logo após seu registro manual.

As especificações técnicas também incluem a geometria da rede de nivelamento (perímetro máximo dos circuitos), mas seu atendimento não foi possível na região amazônica, em função da malha viária reduzida. Parte significativa das linhas de nivelamento no Acre, Amazonas e Roraima não forma circuitos (isto é, são ramais abertos), enquanto em Rondônia, Pará e Mato Grosso os circuitos têm perímetro superior ao limite especificado, determinando tolerâncias de fechamento maiores que as encontradas em outras porções da RAAP. Apesar de não explicitado nas especificações (IBGE, 1983), a tolerância usualmente praticada pelo IBGE para o erro de fechamento dos circuitos da RAAP é $5 \mathrm{~mm}\left[\mathrm{P}_{\mathrm{km}}\right]^{1 / 2}$, sendo $P_{\mathrm{km}}$ o perímetro expresso em quilômetros.

Conforme explanação anterior, não foram utilizadas observações gravimétricas para correção dos dados de nivelamento da RAAP. A justificativa é a ausência, até a década de 90 , desse tipo de observação sobre as RRNN da rede, de forma sistemática. Os extensos levantamentos gravimétricos realizados pelo IBGE na década de 60 concentraram-se na região do ponto datum do sistema geodésico de referência horizontal adotado no Brasil até 2005, o SAD69. Somente a partir de 1991, quando se iniciaram os levantamentos gravimétricos sistemáticos no IBGE, foi possível acompanhar com gravimetria todas as novas linhas de nivelamento. Antes disso, inúmeras instituições realizaram observações gravimétricas, mas nem sempre sobre as RRNN da RAAP (LUZ \& GUIMARÃES, 2001). 
Entre 1948 e 1975 foram executados oito ajustamentos manuais, em blocos justapostos (MATTOS, 1948; BESERRA, 1952; ALENCAR, 1968). Depois disso, iniciou-se a conversão das informações para meio digital, em preparação ao Ajustamento Altimétrico Global Preliminar (AAGP), concluído em 1993 (RIBEIRO \& LUZ, 1991). Uma característica importante a ser ressaltada nos ajustamentos históricos é a aplicação da correção normal-ortométrica $\left(\delta H^{N o r t}\right)$, apesar de não explicitada nos relatórios citados (LUZ, 2008).

Em função da capacidade limitada do programa de ajustamento paramétrico utilizado no AAGP, aqui denominado AJNIV, não foi possível realizar um ajustamento simultâneo da RAAP (RIBEIRO, 1989). Isso deu origem ao particionamento da rede em "macrocircuitos" (MCs), os quais foram ajustados isoladamente, para fornecer altitudes de partida para o ajustamento das subredes internas de cada MC.

No Banco de Dados Geodésicos (BDG), as altitudes resultantes do AAGP tinham datas de referência 01/12/1992 (conjunto principal), 01/12/1993 e posteriores (ramais, subredes isoladas, linhas "novas"). Estas altitudes eram classificadas como "ajustada - alta precisão", quando as respectivas linhas atendiam à tolerância de fechamento de circuitos, ou "ajustada - fora das prescrições", caso contrário.

As altitudes atualmente disponíveis no BDG resultam do reajustamento concluído em 2011 (IBGE, 2011), cuja data de referência, no BDG, é 15/6/2011. Praticamente todas as estações altimétricas do SGB tiveram suas altitudes normais-ortométricas atualizadas nesta data, e os respectivos desvios-padrão foram introduzidos no BDG como informação complementar, substituindo a antiga classificação das altitudes ("ajustada - alta precisão", "ajustada - fora das prescrições", "preliminar"). Além disso, como informação adicional ao usuário do SGB, os desvios-padrão das altitudes das RRNN em ramais (sem controle de fechamento de circuito) não são informados, e o respectivo Relatório de Estação Geodésica (REG) apresenta a correspondente advertência: "A RN pertence a um ramal, isto é, uma linha de nivelamento geométrico que não forma circuito, cujos desníveis, portanto, não podem ser ajustados. Consequentemente, as respectivas altitudes são calculadas mediante simples transporte, sem as estimativas de desvio-padrão". Outra advertência também incorporada aos REGs de RRNN na Região Amazônica diz respeito aos já mencionados circuitos com perímetro muito acima das especificações: "A RN pertence a uma par-

te da Rede Altimétrica cuja falta de alternativas para estabelecimento de circuitos na Região Amazônica leva à impossibilidade de aplicação dos controles de qualidade usuais. Assim, recomendase a adoção de cuidados adicionais na utilização da Rede Altimétrica na área desta RN, tais como o aumento do número de RRNN consideradas no controle do levantamento e a validação dos respectivos desníveis com renivelamento".

\section{Próximo reajustamento da RAAP}

A preparação do próximo reajustamento geral da RAAP foi iniciada em fevereiro de 2016 . Além da incorporação de dados de gravidade em cada desnível observado da RAAP, para fins de determinação de altitudes físicas conforme apresentado na seção 4, foram planejadas outras inovações 
no processo de pré-tratamento das linhas de nivelamento. A principal delas é a automatização da análise topológica da rede, com a identificação dos pontos nodais e a redução internodal das informações de entrada, de modo a eliminar as RRNN intermediárias que não afetam os resultados do reajustamento geral. Assim, a rede de 70 mil pontos é reduzida a menos de 2 mil, simplificando não somente o próprio ajustamento, mas também a verificação preliminar dos circuitos e a análise estatística dos resultados. Posteriormente, os números geopotenciais ajustados das RRNN intermediárias são obtidos em um processo similar à distribuição de erros entre dois pontos conhecidos.

A redução internodal está sendo realizada com uma versão aprimorada da ferramenta computacional IDNOS (LUZ, 2008).

Outra inovação planejada é a vinculação da rede altimétrica no Amapá ao restante da RAAP referido ao DVB-I. Para isso, encontra-se em discussão a aplicação de estratégias avançadas para integração de dados geodésicos sob o esquema geral de solução do problema do valor de contorno da Geodésia (PVCG), como descrito, por exemplo, por Ferreira et al. (2010).

As etapas preparatórias do reajustamento (redução internodal e análise dos fechamentos dos circuitos) demandarão a maior carga de trabalho. A fim de estimar, antecipadamente, os eventuais impactos da incorporação da informação gravimétrica no cálculo das altitudes ajustadas da RAAP, decidiu-se recorrer à rede do reajustamento de 1992/1993 (AAGP), cuja etapa de análise topológica e montagem de circuitos já se encontrava realizada.

Para tanto, a primeira dificuldade a ser vencida foi a mencionada inexistência de observação gravimétrica em grande parte das RRNN da RAAP. Para isso, foi utilizada uma versão adaptada do programa GEOGRID, do sistema Gravsoft (FORSBERG, 2003).

A seguir, as diferenças de geopotencial de cada seção foram calculadas (eq. 5) e acumuladas para cada linha internodal. A partir destas informações internodais, e de uma versão adaptada do programa AJNIVOCT (LUZ, 2008), os números geopotenciais ajustados foram calculados para as 944 RRNN da rede do AAGP, conectadas por 1278 desníveis. A análise desses resultados preliminares ainda se encontra em andamento, mas já foi possível chegar a algumas conclusões interessantes, com base na Figura 5.

Em primeiro lugar, as altitudes normais e as normais-ortométricas, nas estações maregráficas do IAGS, apresentam grande similaridade, validando as informações conceituais de que seria pequena a influência da gravidade observada, em relação à gravidade teórica (normal), nas regiões de baixa altitude.

A manutenção das linhas "rejeitadas" no AAGP, isto é, aquelas que a análise do fechamento de circuitos indicou possuírem problemas, levou a grandes diferenças de altitude em relação ao AAGP (que não sofreu influência de tais linhas). Este resultado vem sendo objeto de cuidadosa análise, de modo a subsidiar corretamente o processo de preparação do novo reajustamento.

Será muito importante a continuação da análise dos resultados deste ensaio, estendendo-os ao interior do país - principalmente às regiões mais altas, onde já foram detectadas diferenças de até $9 \mathrm{~cm}$ entre as altitudes originais do AAGP (normais-ortométricas) e os novos valores (altitudes normais). 


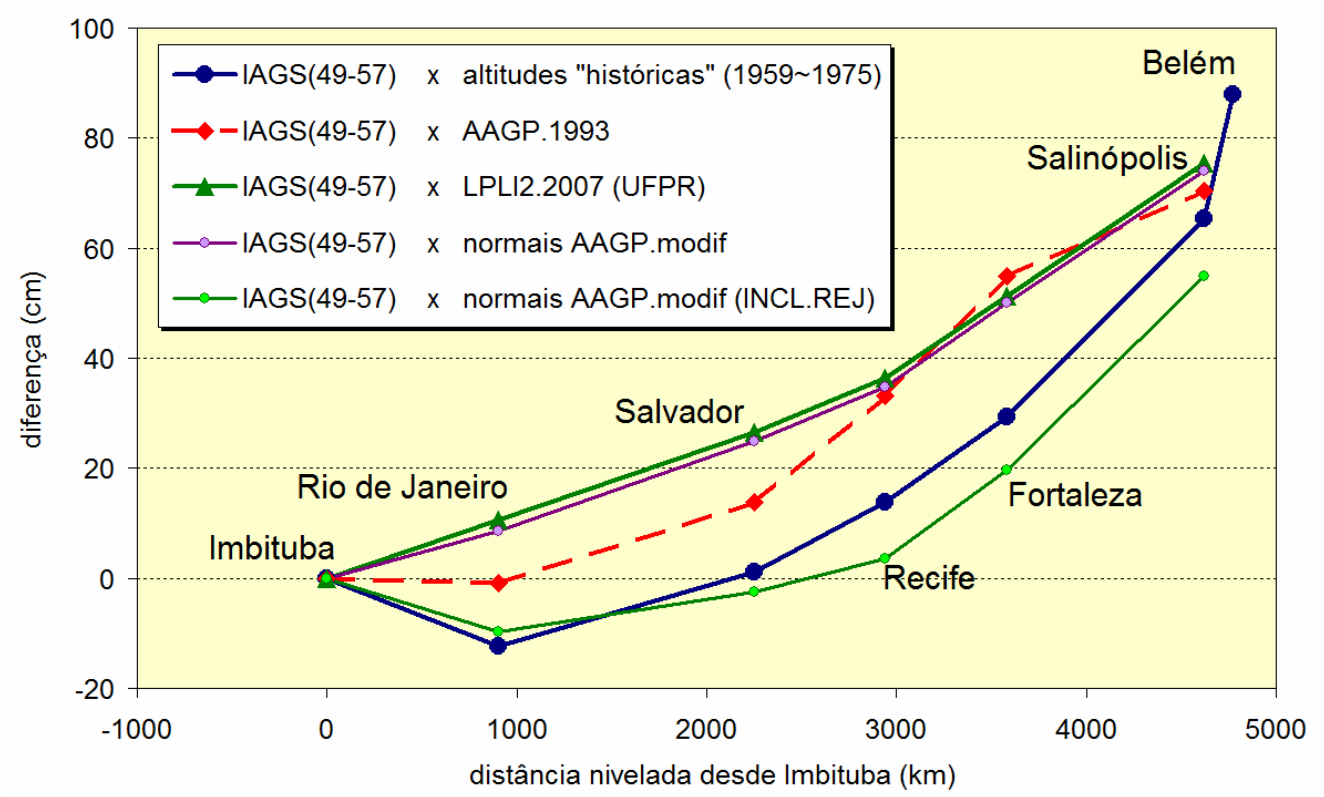

Figura 5. Comparação das altitudes de diversos ajustamentos da RAAP e as altitudes referidas ao NMM local em algumas estações maregráficas do IAGS. Fonte: adaptado de Luz (2008).

\section{Considerações finais}

Foram apresentados conceitos referentes aos sistemas geodésicos de referência vertical e às altitudes físicas, discutindo-se também a forma como tais conceitos vêm sendo realizados no Brasil, e antecipando algumas inovações em preparação para o novo reajustamento geral da Rede Altimétrica de Alta Precisão do SGB.

Espera-se que a conclusão deste reajustamento, prevista para o primeiro semestre de 2017, represente um verdadeiro salto de qualidade das altitudes brasileiras, permitindo o refinamento do Modelo Geoidal, também previsto para 2017. Além disso, o reajustamento da rede também contribuirá para os estudos referentes à integração dos níveis de referência marinhos e terrestres, no contexto do Comitê Especializado da CONCAR criado especificamente para tratar deste tema. 
Referências

ALENCAR, J.C.M. Sistema Nacional de Nivelamento de 1a Ordem. In: CONFERÊNCIA NACIONAL DE GEOCIÊNCIAS, v.1, Rio de Janeiro. Anais... Rio de Janeiro: IBGE, 1968. Disponível em: <http://artigos.ibge.gov.br/artigos-home/geodesia/ate-1989.html>. Acesso em: 29 jan. 2016.

ALENCAR, J.C.M. Datum Altimétrico Brasileiro. Cadernos de Geociências v.5, p. 69-73, 1990. Disponível em: <http://artigos.ibge.gov.br/artigos-home/geodesia/1999-1989.html>. Acesso em: 29 jan. 2016.

BESERRA, H. Instruções para Nivelamentos de Precisão. Rio de Janeiro: CNG, 1948. 29 p. (Biblioteca Geográfica Brasileira, Série C, n. 4).

BESERRA, H. Ajustamento da Rede de Nivelamento. Rio de Janeiro: CNG, 1952. (Biblioteca Geográfica Brasileira, Série B, n. 11). Disponível em: <http://artigos.ibge.gov.br/artigoshome/geodesia/ate-1989.html>. Acesso em: 29 jan. 2016.

BOSCH, W. The Sea Surface Topography and its Impact to Global Height System Definition. Vertical Reference Systems (IAG Symposia, v.124). Berlin: Springer, p. 225-230, 2002.

BURŠA, M.; KENYON, S.; KOUBA, J.; RADEJ, K.; VATRT, V.; VOJTÍŠKOVÁ, M.; ŠIMEK, J. World Height System Specified by Geopotential at Tide Gauge Stations. Vertical Reference Systems (IAG Symposia, v.124). Berlin: Springer, p. 291-296, 2002.

DREWES, H.; FORTES, L.P.S.; HOYER, M.; LUZ, R.T. The Vertical Reference Frame for the Americas - the SIRGAS 2000 GPS Campaign. Vertical Reference Systems (IAG Symposia, v.124). Berlin: Springer, p. 302-305, 2002a.

DREWES, H.; SÁNCHEZ, L.; BLITZKOW, D.; FREITAS, S. Scientific Foundations of the SIRGAS Vertical Reference System. Vertical Reference Systems (IAG Symposia, v.124). Berlin: Springer, p. 297-301, 2002b.

FEATHERSTONE, W. E.; KIRBY, J. F.; HIRT, C.; FILMER, M. S.; CLAESSENS, S.J.; BROWN, N.J.; HU, G.; JOHNSTON, G.M. The AUSGeoid09 model of the Australian Height Datum. Journal of Geodesy v.85, n.3, p. 133-150, 2011.

FERREIRA, V.G.; DE FREITAS, S.R.C.; HECK, B. Determination of the Geopotential Difference of the Brazilian Vertical Datum with Respect to the Earth Gravity Model 2008. Revista Brasileira de Cartografia v.62, n.3, p. 467-477, 2010.

FORSBERG, R. An overview manual for the GRAVSOFT Geodetic Gravity Field Modelling Programs. Incl. comm. by C. C. Tscherning and P. Knudsen (DNSC). DRAFT - 1.ed. 2003.

FREITAS, S.R.C.; BLITZKOW, D. Altitudes e Geopotencial. IGeS Bulletin, International Geoid Service v.9, Special Issue, p. 47-61, 1999.

GEMAEL, C. Introdução à Geodésia Física. 1a ed. (reimpr.). Curitiba: UFPR, 2002.

GRAFAREND, E.W. What is a Geoid?. VANÍCEK, P.; CHRISTOU, N.T. (Ed.). Geoid and Its Geophysical Interpretations. Boca Raton: CRC Press, 1994, p. 3-32. 
HECK, B. Problems in the Definition of Vertical Reference Frames. V Hotine-Marussi Symposium on Mathematical Geodesy (IAG Symposia, v.127). Berlin: Springer, p. 164-173, 2004.

HEISKANEN, W.A.; MORITZ, H. Physical Geodesy. San Francisco: Freeman, 1967. 364 p.

HWANG, C.; HSIAO, Y.-S. Orthometric corrections from leveling, gravity, density and elevation data: a case study in Taiwan. Journal of Geodesy v.77, n.5-6, p. 279-291, 2003.

IAG. Report of the Ad-hoc Group on an International Height Reference System (IHRS). 2015a. Disponível em: <http://iag.dgfi.tum.de/fileadmin/IAG-docs/Travaux2015/ 26_Concept_IHRS_Actions_Proposal_V28.pdf>.Acesso em: 29 jan. 2016.

IAG. IAG Resolution ( $\left.N^{\circ} .1\right)$ for the definition and realization of an International Height Reference System (IHRS). 2015b. Disponível em: <http://iag.dgfi.tum.de/fileadmin/IAGdocs/IAG_Resolutions_2015.pdf>. Acesso em: 29 jan. 2016.

IBGE. Resolução do Presidente (R. PR) n. 22 de 21/julho/1983. Especificações e Normas Gerais para Levantamentos Geodésicos. Boletim de Serviço, Rio de Janeiro: IBGE, n.1602, 1983. Disponível em: <http://www.ibge.gov.br/home/geociencias/ geodesia/default_normas.shtm>. Acesso em: 29 jan. 2016.

IBGE. Nivelamento Geodésico - Manual de Instruções. 2a. ed. Fortaleza: IBGE, 1985.

IBGE. Relatório Final - Grupos de Trabalho I e II do Projeto SIRGAS. Rio de Janeiro, 1997.

IBGE. Ajustamento Simultâneo da Rede Altimétrica de Alta Precisão do Sistema Geodésico Brasileiro 2011. Disponível em <http://www.ibge.gov.br/home/geociencias/geodesia/altimetrica.shtm>. Acesso em: 24 set. 2015.

IBGE. Relatório de Monitoramento da Variação do Nível Médio do Mar nas Estações da Rede Maregráfica Permanente para Geodésia, 2001-2012. Rio de Janeiro: IBGE, 2013. 46p. Disponível <http://www.ibge.gov.br/home/geociencias/geodesia/rmpg/default_rmpg_int.shtm>. Acesso em: 24 set. 2015.

JEKELI, C. Heights, the Geopotential, and Vertical Datums (Report, Dept. of Civ. and Env. Eng. and Geodetic Science, n. 459). Columbus: OSU, 2000. 34 p. Disponível em: <http://www.ceegs.ohio-state.edu/gsreports/>. Acesso em: 15 ago. 2005.

LUZ, R.T.; GUIMARÃES, V.M. Realidade e Perspectivas da Rede Altimétrica de Alta Precisão do Sistema Geodésico Brasileiro. In: II COLÓQUIO BRASILEIRO DE CIÊNCIAS GEODÉSICAS, Curitiba. Anais... Curitiba, 2001. Disponível em: <http://artigos.ibge.gov.br/artigoshome/geodesia/2004-2000.html>. Acesso em: 29 jan. 2016.

LUZ, R.T.; GUIMARÃES, V. M. Dez Anos de Monitoramento do Nível do Mar no IBGE. In: III COLÓQUIO BRASILEIRO DE CIÊNCIAS GEODÉSICAS, Curitiba. Anais... Curitiba, 2003. Disponível em: <http://artigos.ibge.gov.br/artigos-home/geodesia/2004-2000.html>. Acesso em: 29 jan. 2016.

LUZ, R.T. Estratégias para Modernização da Componente Vertical do Sistema Geodésico Brasileiro e sua Integração ao SIRGAS. Tese (Doutorado). Curitiba: CPGCG/UFPR, 2008. 
179p. Disponível em: <http://dspace.c3sl. ufpr.br/dspace/handle/1884/17075>. Acesso em: 28 set. 2013.

MATTOS, A.H. Ajustamento Preliminar de uma Rede de Nivelamento. Rio de Janeiro: CNG, 1948. (Biblioteca Geográfica Brasileira, Série B, n. 7). Disponível em: <http://artigos.ibge.gov.br/artigos-home/geodesia/ate-1989.html>. Acesso em: 29 jan. 2016.

RIBEIRO, G.P. Ajustamento Altimétrico desenvolvido através do Método das Equações de Observação e com Análise Estatística dos Resultados. Dissertação (Mestrado). Curitiba: UFPR, 1989. $245 \mathrm{f}$.

RIBEIRO, G.P.; LUZ, R.T. Resultados Preliminares do Ajustamento Global da Rede Altimétrica de Alta Precisão do Sistema Geodésico Brasileiro. Congresso Brasileiro de Cartografia. Anais, v.1. São Paulo: SBC, p. 155-162, 1991. Disponível em: <http://artigos.ibge.gov.br/artigos-home/geodesia/1999-1989. html>. Acesso em: 29 jan. 2016.

SÁNCHEZ, L. Reporte 2005, SIRGAS-GTIII. Disponível em: <http://www.sirgas. org/fileadmin/docs/Boletines/Bol09/23_Sanchez_ReporteGTIII.pdf>. Acesso em: 29 jan. 2016.

SÁNCHEZ, L. Towards a vertical datum standardisation under the umbrella of Global Geodetic Observing System. Journal of Geodetic Science v.2, n.4, p. 325-342, 2012.

SIRGAS. Reporte 2010-2011. Disponível em: <http://www.sirgas.org/fileadmin/ docs/Boletines/Boletin_SIRGAS_No_16.pdf>. Acesso em: 29 jan. 2016.

SJÖBERG, L.E. New solutions for the geoid potential WO and the Mean Earth Ellipsoid dimensions. Journal of Geodetic Science v.3, n.4, p. 258-265, 2013.

TORGE, W. Geodesy. 3th ed. (rev. e ext.). Berlin: Walter de Gruyter, 2001, xv, 416 p.

VANÍCEK, P. Physical Geodesy. Saint John: University of New Brunswick, 1976. 176 p.

VANÍCEK, P.; KRAKIWSKY, E.J. Geodesy: the Concepts. 2nd ed. Amsterdam: Elsevier, 1986. $697 \mathrm{p}$.

ZILKOSKI, D.B.; RICHARDS, J.H.; YOUNG, G.M. Results of the General Adjustment of the North American Vertical Datum of 1988. Surveying and Land Information Systems v.52, n.3, p. 133-149, 1992. 